\title{
Using noise to break the noise barrier in circuits
}

\author{
Ferran Martorell ${ }^{a}$, Mark D. McDonnell ${ }^{b}$, Antonio Rubio ${ }^{a}$ and Derek Abbott $^{b}$ \\ ${ }^{a}$ High Performance IC Design Group, Univ. Politècnica de Catalunya (DEE), Ed.C-4 Jordi \\ Girona 1-3 08034, Barcelona, Spain; \\ ${ }^{b}$ Centre for Biomedical Engineering (CBME) and School of Electrical \& Electronic \\ Engineering, The University of Adelaide, SA 5005, Adelaide, Australia
}

\begin{abstract}
Technology advances tend to reduce minimum dimensions and source voltages to maintain scaling rules. Both scaling trends make noise more critical, reduce yield and increase device parameter fluctuations. This paper presents an statistical model that permits the study of noise and parameter deviations on gates. Using this model stochastic resonance (SR) is studied both in single devices and arrays for subthreshold and suprathreshold input signals. The SR is measured by the signal-to-noise ratio (SNR) in the time domain and a modified SNR is proposed to take into account all the effects induced by noise in gates. With this measure subthreshold and suprathreshold SR is reviewed. Finally, a discussion of the possibility of considering noise a part of the electronic circuits is presented, suggesting that it could be a solution to some of the emerging problems in future nanotechnologies.
\end{abstract}

Keywords: noise, suprathreshold stochastic resonance, stochastic resonance, nonlinear circuits, low SNR signal processing

\section{INTRODUCTION}

Since the invention of the microchip in the 60's, progress in microelectronics technology has relied on advances in miniaturization and precision in manufacturing processes. These trends have provided present-day technology with chips composed of $10^{8}$ transistors, clock frequencies above $1 \mathrm{GHz}$ and gate dimensions below $100 \mathrm{~nm}$. However, the impressive achievements in microelectronics technology has produced several problems, which threaten the ever increasing need for technology performance. Technologies near a $50 \mathrm{~nm}$ gate length suffer severe reduction in yield and performance, and predictions for smaller technologies further increase the concern about these problems. ${ }^{1} \quad$ Future nanoelectronic technologies will have severe problems in maintaining yield, parameter variation along the chip and noise immunity - as signal levels reduce to meet power consumption requirements. This situation will require a different approach to electronic design, both for coping with these emerging problems and future devices. In any case defects ${ }^{2}$ and noise ${ }^{3}$ (either internal or external) will be a key factor for next generation technologies. A possible solution is the optimal combination of imperfect components paradigm expounded by Johnson et al. ${ }^{4}$ However, in this paper we will focus on the exploitation of a mechanism called stochastic resonance (SR).

In contrast to the ideal world of freedom from semiconductor defects, controlled fluctuations and a perfectly predictable environment, demanded by the electronics industry, biological systems have developed their working basis upon a system full of defects with great variability and noise. Biological systems have evolved to be either immune to noise or to take advantage of it. Several studies show how biological sensory systems use noise to improve their sensitivity, ${ }^{5,6}$ how sensory neurons deal with information in a noisy environment ${ }^{7}$ where signalto-noise ratio (SNR) can be as low as $0 \mathrm{~dB}$, and even how neurons can use noise to achieve a better working dynamic range. ${ }^{8}$ The principles ruling biological systems are still far from being understood, however the study

Further author information: (Send correspondence to F.M.)

F.M.: E-mail: ferranm@eel.upc.es, Telephone: +34-93-4017-485

M.D.M.: E-mail: mmcdonne@eleceng.adelaide.edu.au, Telephone: +61-8-8303-6296

A.R: E-mail: rubio@eel.upc.es, Telephone: +34-93-4017-485

D.A.: E-mail: dabbott@eleceng.adelaide.edu.au, Telephone: +61-8-8303-5748 
of stochastic resonance appears to be bridging the gap. Stochastic resonance is a phenomenon by which noise, combined with a non-linear system, can improve the system performance. For example, in measurement, adding noise and averaging afterwards produces a resolution improvement. ${ }^{9}$ Moreover, in ADC/DAC applications dithering also permits extra resolution and noise may avoid undesired instabilities. ${ }^{10}$ Vibrations can be put to use and rectified to supply energy. ${ }^{11}$ SR has proved to be of utility in detection of weak signals in noisy environments as reported by a number of researchers. ${ }^{12-14}$ Recently several applications related to SR have been presented e.g. in nanotube FETs used to improve signal detection in nanoantennas, ${ }^{15}$ in a receiver used to retrieve subthreshold digital data ${ }^{16}$ or even in a gravitational wave interferometer to increase its sensitivity. ${ }^{17}$ Stocks et al. have applied suprathreshold stochastic resonance to cochlear implant technology ${ }^{18}$ and Harmer et al. have investigated it in artificial insect vision models. ${ }^{19}$

Most studies, however, consider an ideal threshold gate as a non-linear system - however, this is not a realistic model for electronic gates. In this work we will consider a soft limiter as a more realistic model of an electronic circuit element to explore the phenomena of subthreshold and suprathreshold SR. We develop a model for general circuit input-output functions suitable for studying noise effects and variations on circuits parameters. The function and error for single gates and systems based on an array of gates is presented. Once the model is presented we check its predictions studying SR considering both subthreshold and suprathreshold input signals using the signal-to-noise ratio in time domain. Finally we modify this measurement to obtain a better measure of system performance suitable for non-linear electronic systems.

\section{MODEL}

As stated in the introduction, noise and parameter deviations will be the main limiting factors in next generation of microelectronic systems. In this section we model a basic cell permitting the study of both factors. We concentrate on a single cell considering a generic static function, $g(\cdot)$, with an input consisting of an input signal, $x$, and added noise, $\eta_{i}$, producing an output, $y_{i}$, as Figure 1 (left) shows. Note that $\eta_{i}$ permits the modeling of the main noise effects in an electronic system, as most electronic noise can be considered to be additive. Process variation is modeled in the $g(\cdot)$ function, where we introduce the effects of any possible deviation. In this work we consider two different functions: (i) a hard limiter defined by its threshold value, $b$, and its output levels, $a_{h}$ and $a_{l}$, and (ii) a soft limiter defined by four parameters: $b_{h}$ and $b_{l}$ determining the linear transition from both possible states and the output levels, $a_{h}$ and $a_{l}$. Figure 2 depicts both functions.

Once we have the single gate model we extend it to an array system as it adds the possibility of suprathreshold SR. ${ }^{20,21}$ Figure 1 shows the structure for the array system. In it we consider an array of $n$ gates in parallel with a common input signal, $x$, and additive noise $\eta_{i}$, without loss of generality. The outputs $y_{i}$ are summed together and then scaled to produce the system output, $\bar{y}$.

To be able to design an electronic system from individual gates, it is necessary to know the gate function and its error. So the model gives both answers in a statistical fashion. The input-output function of the gate can be described by the expected value of the gate output conditioned on the input signal, $E\{y \mid x\}$, and the error by the variance of the output given the input signal, $\sigma_{y \mid x}^{2}$. Both values depend on the probability density function (pdf) of the output variable as Equations (1) and (2) show,

$$
\begin{gathered}
E\{y \mid x\}=\int_{-\infty}^{\infty} y f_{y}(y \mid x) d y \\
\sigma_{y \mid x}^{2}=\int_{-\infty}^{\infty}(y-E\{y \mid x\})^{2} f_{y}(y \mid x) d y .
\end{gathered}
$$




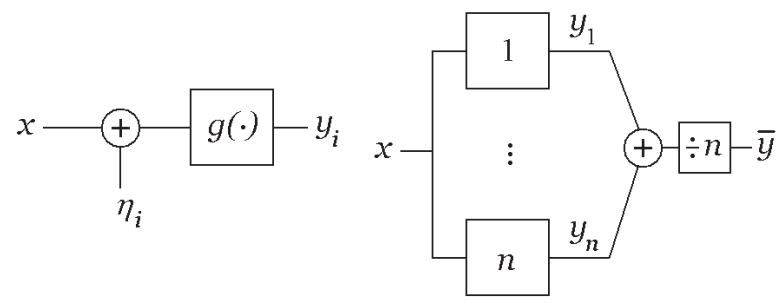

Figure 1. Left: Simple schematic structure of the system under study. Two random variables (RV) $x$ and $\eta$ as input and an output RV $y_{i}=g\left(x+\eta_{i}\right)$. Here, $g(\cdot)$ stands for any transfer function. Right: Array system with $n$ gates combining all individual outputs $y_{i}$ by averaging them to produce the total output $\bar{y}$.

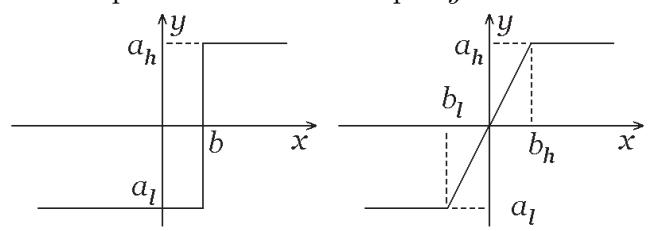

Figure 2. Functions for the hard limiter (left) and soft limiter (right) with their parameters defined.

\subsection{Modeling single gates}

In this section the model for single gates is presented. The general pdfs for both functions considering a general noise distribution are presented. The description considers the interaction between noise and function as the principal characteristic. This yields a conditioned pdf to the input signal and all the parameters that define each function. However to simplify notation we only write the input signal dependence explicitly. Once the general formulation is presented we apply the model to uniform and Gaussian noise distributions, as they are the easiest to mathematically describe and commonly occur in natural processes.

\subsubsection{Generic noise distribution}

The derivation of the model for a general noise distribution is presented for both functions. The gate function, $g(\cdot)$ is rewritten in terms of the noise signal, $\eta$, so the resulting formulation shows the output dependance on all variables of interest.

\section{Hard limiter with random threshold values}

The ideal comparator or hard limiter is a non-linear function defined as (written in terms of the noise variable)

$$
y_{i}=g(\eta)= \begin{cases}a_{l} & b-x \leq \eta \\ a_{h} & b-x>\eta\end{cases}
$$

According to this function the output value, $y_{i}$, can take only two discrete values depending on whether the input is higher or lower than its threshold. In this case we can consider an equivalent threshold defined by the actual threshold minus the input signal $(b-x)$. So its pdf for a general noise is

$$
f_{y_{i} \mid x}\left(y_{i} \mid x\right)=F_{\eta}(b-x) \delta\left(y_{i}-a_{l}\right)+\left(1-F_{\eta}(b-x)\right) \delta\left(y_{i}-a_{h}\right) .
$$

Soft Limiter function with random threshold values

Electronic non-linear comparator and electronic digital gates in general have a behavior that can be described in terms of a transition between two stable output values. This general behavior can be modeled by a soft limiter function as a first order model. In this case and acting as before the function $g(\cdot)$ is

$$
y_{i}=g(\eta)=\left\{\begin{array}{lc}
a_{l} & \eta<b_{l}-x \\
\frac{a_{h}-a_{l}}{b_{h}-b_{l}}\left(\eta-b_{l}+x\right)+a_{l} & b_{l}-x \leq \eta \leq b_{h}-x \\
a_{h} & \eta>b_{h}-x .
\end{array}\right.
$$




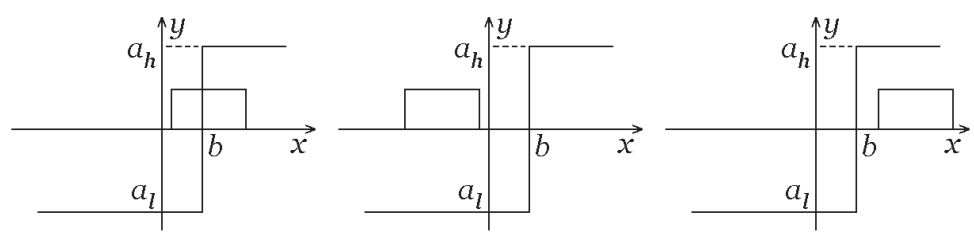

Figure 3. Possible situations for the hard limiter circuit as described in Equation (7).

Now the gate has two discrete values high $\left(a_{h}\right)$ and low $\left(a_{l}\right)$ and any value between them. Its pdf is then described as

$$
\begin{aligned}
f_{y_{i}}\left(y_{i} \mid x\right)=\frac{b_{h}-b_{l}}{a_{h}-a_{l}} f_{\eta}\left(\frac{b_{h}-b_{l}}{a_{h}-a_{l}}\left(y_{i}-a_{l}\right)+b_{l}-x\right)\left(u\left(y_{i}-a_{l}\right)-u\left(y_{i}-a_{h}\right)\right)+ \\
+F_{\eta}\left(b_{l}-x\right) \delta\left(y_{i}-a_{l}\right)+\left(1-F_{\eta}\left(b_{h}-x\right)\right) \delta\left(y_{i}-a_{h}\right) .
\end{aligned}
$$

\subsubsection{Uniform noise}

The simplest statistical distribution is the uniform distribution. This distribution has little practical interest as it is not present in physical processes, but its mathematical simplicity permits a complete derivation giving closed form solutions. This fact makes it interesting as the gate function is easily understandable. However as the noise cannot reach the whole input range, the description is necessarily done piecewise according to the region in which the noise is present. We consider a uniform noise in the range $-A / 2$ to $A / 2$ with amplitude $1 / A$.

\section{Hard limiter with random threshold values}

The hard limiter combined with uniform noise has three possible working regions depending on the relation between noise values and the equivalent threshold function. These situations are depicted in Figure 3. Two of them are symmetric whenever there is no level crossings giving a constant output value. The last situation and most interesting appears when the noise produce transitions from one state to the other. The pdf of the output conditioned to the input is

$$
f_{y_{i}}\left(y_{i} \mid x\right)=\left\{\begin{array}{cc}
\frac{1}{A}\left[\left(\frac{A}{2}+b-x\right) \delta\left(y_{i}-a_{l}\right)+\left(\frac{A}{2}-(b-x)\right) \delta\left(y_{i}-a_{h}\right)\right] & \begin{array}{c}
-\frac{A}{2}<b-x<\frac{A}{2}, \\
a_{l} \leq y_{i} \leq a_{h} \\
b-x \geq \frac{A}{2}, y_{i}=a_{l} \\
\delta\left(y_{i}-a_{l}\right)
\end{array} \\
\delta\left(y_{i}-a_{h}\right) & b-x \leq-\frac{A}{2}, y_{i}=a_{h} .
\end{array}\right.
$$

Once the pdf is obtained we calculate the expected value and variance using Equations (1) and (2).

$$
\begin{gathered}
E\left\{y_{i} \mid x\right\}=\left\{\begin{array}{cc}
\frac{a_{h}+a_{l}}{2}+\frac{a_{h}-a_{l}}{A}(x-b) & -\frac{A}{2}<b-x<\frac{A}{2} \\
a_{l} & b-x \geq \frac{A}{2} \\
a_{h} & b-x \leq-\frac{A}{2}
\end{array}\right. \\
\sigma_{y_{i} \mid x}^{2}=\left\{\begin{array}{cc}
\frac{\left(a_{h}-a_{l}\right)^{2}}{A^{2}}\left[\left(\frac{A}{2}\right)^{2}-(x-b)^{2}\right] & -\frac{A}{2}<b-x<\frac{A}{2} \\
0 & b-x \geq \frac{A}{2} \\
0 & b-x \leq-\frac{A}{2} .
\end{array}\right.
\end{gathered}
$$

Soft limiter function with random threshold values

In this case we have six possible states (four of them symmetrical). Figure 4 depicts them. One state where 

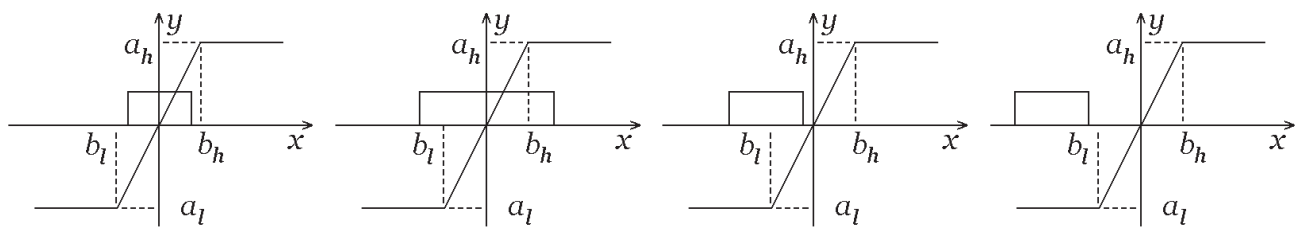

Figure 4. From left to right cases 1, 2, 3, and 5 from Eq. (10).

noise values only cover the linear section, one where they cover the whole linear section of the soft limiter and more in both sides, two states where only a part of the linear section is covered and two where no noise values reach the linear section. According to them the cell pdf is written as

$$
f_{y_{i}}\left(y_{i} \mid x\right)=\left\{\begin{array}{c}
\frac{1}{A} \frac{b_{h}-b_{l}}{a_{h}-a_{l}} \\
\frac{1}{A}\left[\frac{b_{h}-b_{l}}{a_{h}-a_{l}}+\left(\frac{A}{2}+b_{l}-x\right) \delta\left(y_{i}-a_{l}\right)+\left(\frac{A}{2}-\left(b_{h}-x\right)\right) \delta\left(y_{i}-a_{h}\right)\right] \\
\frac{1}{A}\left[\frac{b_{h}-b_{l}}{a_{h}-a_{l}}+\left(\frac{A}{2}+b_{l}-x\right) \delta\left(y_{i}-a_{l}\right)\right] \\
\frac{1}{A}\left[\frac{b_{h}-b_{l}}{a_{h}-a_{l}}+\left(\frac{A}{2}-\left(b_{h}-x\right)\right) \delta\left(y_{i}-a_{h}\right)\right] \\
\delta\left(y_{i}-a_{l}\right) \\
\delta\left(y_{i}-a_{h}\right)
\end{array}\right.
$$

$$
\begin{gathered}
-\frac{A}{2} \geq b_{l}-x \text { and } b_{h}-x \geq \frac{A}{2}, \\
a_{\min } \leq y_{i} \leq a_{\max } \\
-\frac{A}{2} \leq b_{l}-x \text { and } b_{h}-x \leq \frac{A}{2}, \\
a_{l} \leq y_{i} \leq a_{h} \\
-\frac{A}{2}<b_{l}-x \text { and } b_{h}-x>\frac{A}{2} \\
\text { and } b_{l}-x<\frac{A}{2}, a_{l} \leq y_{i} \leq a_{\max } \\
-\frac{A}{2}>b_{l}-x \text { and } b_{h}-x<\frac{A}{2} \\
\text { and } b_{h}-x>-\frac{A}{2}, a_{\min } \leq y_{i} \leq a_{h} \\
b_{l}-x \geq \frac{A}{2}, y_{i}=a_{l} \\
b_{h}-x \leq-\frac{A}{2}, y_{i}=a_{h},
\end{gathered}
$$

where

$$
a_{\max }=\frac{a_{h}-a_{l}}{b_{h}-b_{l}}\left(\frac{A}{2}+x-b_{l}\right)+a_{l}, \quad a_{\min }=\frac{a_{h}-a_{l}}{b_{h}-b_{l}}\left(-\frac{A}{2}+x-b_{l}\right)+a_{l},
$$

are the maximum and minimum values that variable $y_{i}$ can take when $a_{h}$ and $a_{l}$ are out of reach.

In the same way as before, we work out the expected value and variance for this distribution obtaining the gate function and associated error,

$$
E_{y_{i}}\left\{y_{i} \mid x\right\}=\left\{\begin{array}{cc}
\frac{a_{h}-a_{l}}{b_{h}-b_{l}}\left(x-b_{l}\right)+a_{l} & -\frac{A}{2} \geq b_{l}-x \text { and } b_{h}-x \geq \frac{A}{2} \\
\frac{a_{h}+a_{l}}{2}+\frac{a_{h}-a_{l}}{A}\left(x-\frac{b_{h}+b_{l}}{2}\right) & -\frac{A}{2} \leq b_{l}-x \text { and } b_{h}-x \leq \frac{A}{2} \\
\frac{1}{2 A} \frac{a_{h}-a_{l}}{b_{h}-b_{l}}\left(x-b_{l}+\frac{A}{2}\right)^{2}+a_{l} & -\frac{A}{2}<b_{l}-x \text { and } b_{h}-x>\frac{A}{2} \\
a_{h}-\frac{1}{2 A} \frac{a_{h}-a_{l}}{b_{h}-b_{l}}\left(x-b_{h}-\frac{A}{2}\right)^{2} & \text { and } b_{l}-x<\frac{A}{2} \\
a_{l} & \begin{array}{c}
\frac{A}{2}>b_{l}-x \text { and } b_{h}-x<\frac{A}{2} \\
\text { and } b_{h}-x>-\frac{A}{2}
\end{array} \\
a_{h} & b_{l}-x \geq \frac{A}{2} \\
b_{h}-x \leq-\frac{A}{2},
\end{array}\right.
$$




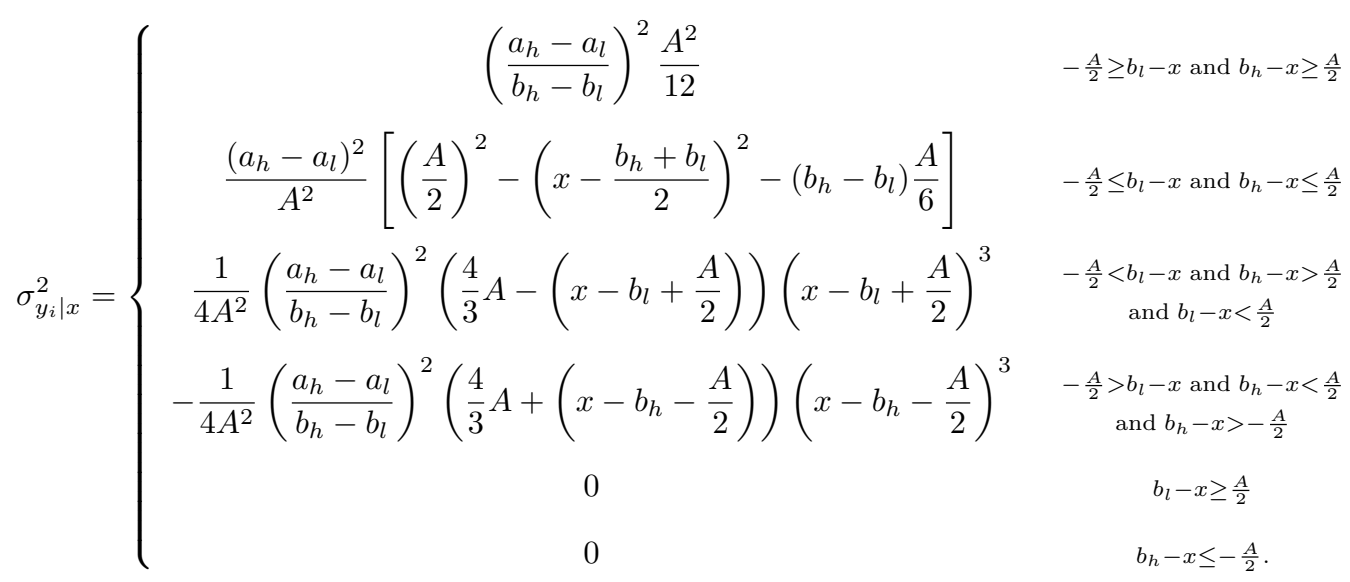

\subsubsection{Gaussian noise}

Gaussian noise with mean $\mu$ and standard deviation $\sigma$ is considered in this case. This distribution is the most common in real processes. In fact the central limit theorem states that the sum of any random variables converges to a Gaussian distribution, as the sum increases. In electronic chips there are many sources of perturbation affecting the devices and so we assume the resulting pdf is Gaussian or normal. ${ }^{22}$ This simplification ignores low-frequency $1 / f$ noise, which can be considered in future studies. The Gaussian pdf is,

$$
f_{\eta}(\eta)=\frac{1}{\sigma \sqrt{2 \pi}} \mathrm{e}^{-\frac{(\eta-\mu)^{2}}{2 \sigma^{2}}},
$$

with probability cumulative function

$$
F_{\eta}(\eta)=\frac{1}{2}\left[1+\operatorname{erf}\left(\frac{\eta-\mu}{\sqrt{2} \sigma}\right)\right]
$$

\section{Hard limiter with random threshold values}

In the same manner, we obtain the pdf for the gate output showing the dependance on input signal and all parameters

$$
f_{y_{i}}\left(y_{i} \mid x\right)=\left[\frac{1}{2}+\frac{1}{2} \operatorname{erf}\left(\frac{b-x-\mu}{\sqrt{2} \sigma}\right)\right] \delta\left(y_{i}-a_{l}\right)+\left[\frac{1}{2}-\frac{1}{2} \operatorname{erf}\left(\frac{b-x-\mu}{\sqrt{2} \sigma}\right)\right] \delta\left(y_{i}-a_{h}\right) .
$$

From it the gate function

$$
E_{y_{i}}\left\{y_{i} \mid x\right\}=\frac{a_{h}+a_{l}}{2}-\frac{a_{h}-a_{l}}{2} \operatorname{erf}\left(\frac{b-x-\mu}{\sqrt{2} \sigma}\right)
$$

and its error

$$
\sigma_{y_{i} \mid x}^{2}=\frac{\left(a_{h}-a_{l}\right)^{2}}{4}\left(1-\operatorname{erf}^{2}\left(\frac{b-x-\mu}{\sqrt{2} \sigma}\right)\right)
$$

are calculated.

Soft limiter function with random threshold values

And finally the soft limiter with Gaussian white noise has an output pdf as

$$
\begin{gathered}
f_{y_{i}}\left(y_{i} \mid x\right)=\frac{b_{h}-b_{l}}{a_{h}-a_{l}} \frac{1}{\sigma \sqrt{2 \pi}} \mathrm{e}^{-\frac{\left(\frac{b_{h}-b_{l}}{a_{h}-a_{l}}\left(y_{i}-a_{l}\right)+b_{l}-x-\mu\right)^{2}}{2 \sigma^{2}}}\left(u\left(y_{i}-a_{l}\right)-u\left(y_{i}-a_{h}\right)\right)+ \\
+\left[\frac{1}{2}+\frac{1}{2} \operatorname{erf}\left(\frac{b_{l}-x-\mu}{\sqrt{2} \sigma}\right)\right] \delta\left(y_{i}-a_{l}\right)+\left[\frac{1}{2}-\frac{1}{2} \operatorname{erf}\left(\frac{b_{h}-x-\mu}{\sqrt{2} \sigma}\right)\right] \delta\left(y_{i}-a_{h}\right) .
\end{gathered}
$$


In this case it is not possible to write a close form solution for all the integrals involved. They yield gate function and error, by numerical solution,

$$
\begin{aligned}
& E_{y_{i}}\left\{y_{i} \mid x\right\}=\int_{a_{l}}^{a_{h}} \frac{b_{h}-b_{l}}{a_{h}-a_{l}} \frac{y_{i}}{\sigma \sqrt{2 \pi}} \mathrm{e}^{-\frac{\left(\frac{b_{h}-b_{l}}{a_{h}-a_{l}}\left(y_{i}-a_{l}\right)+b_{l}-x-\mu\right)^{2}}{2 \sigma^{2}}} d y_{i}+ \\
& +\frac{a_{h}+a_{l}}{2}+\frac{a_{l}}{2} \operatorname{erf}\left(\frac{b_{l}-x-\mu}{\sqrt{2} \sigma}\right)-\frac{a_{h}}{2} \operatorname{erf}\left(\frac{b_{h}-x-\mu}{\sqrt{2} \sigma}\right), \\
& \sigma_{y_{i} \mid x}^{2}=\int_{a_{l}}^{a_{h}} \frac{b_{h}-b_{l}}{a_{h}-a_{l}} \frac{y_{i}^{2}}{\sigma \sqrt{2 \pi}} \mathrm{e}^{-\frac{\left(\frac{b_{h}-b_{l}}{a_{h}-a_{l}}\left(y_{i}-a_{l}\right)+b_{l}-x-\mu\right)^{2}}{2 \sigma^{2}}} d y_{i}+ \\
& -\left[\int_{a_{l}}^{a_{h}} \frac{b_{h}-b_{l}}{a_{h}-a_{l}} \frac{y_{i}}{\sigma \sqrt{2 \pi}} \mathrm{e}^{-\frac{\left(\frac{b_{h}-b_{l}}{a_{h}-a_{l}}\left(y_{i}-a_{l}\right)+b_{l}-x-\mu\right)^{2}}{2 \sigma^{2}}} d y_{i}\right]^{2}-
\end{aligned}
$$

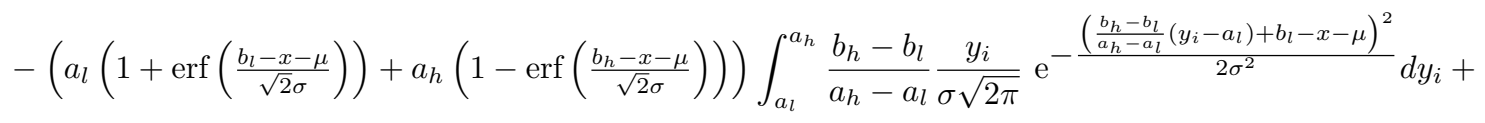

$$
\begin{aligned}
& +\frac{\left(a_{h}-a_{l}\right)^{2}}{4}-\frac{\left(a_{h} \operatorname{erf}\left(\frac{b_{h}-x-\mu}{\sqrt{2} \sigma}\right)-a_{l} \operatorname{erf}\left(\frac{b_{l}-x-\mu}{\sqrt{2} \sigma}\right)\right)^{2}}{4}+\frac{a_{h} a_{l}}{2}\left(\operatorname{erf}\left(\frac{b_{h}-x-\mu}{\sqrt{2} \sigma}\right)-\operatorname{erf}\left(\frac{b_{l}-x-\mu}{\sqrt{2} \sigma}\right)\right) .
\end{aligned}
$$

\subsubsection{Comparison between noise distributions}

Figure 5 shows the function and error performed by a single gate driven by uniform noise. Figure 6 is for Gaussian noise. We have considered a system with symmetrical source levels $\left(a_{h}=0.5 \mathrm{~V}\right.$ and $\left.a_{l}=-0.5 \mathrm{~V}\right)$ with threshold $b=0 \mathrm{~V}$ for the hard limiter and $b_{h}=0.25 \mathrm{~V}$ and $b_{l}=-0.25 \mathrm{~V}$ for the soft limiter. The plots show the function for an input range equal to the source range $\left(x=\left[a_{l}, a_{h}\right]\right)$ and with different standard deviations $(\sigma)$ for the noise considered in the input ranging from $0.1 \mathrm{~V}_{\mathrm{rms}}$ to $3.0 \mathrm{~V}_{\mathrm{rms}}$.

It is interesting to see how noise converts two different circuits into the same response. For both distributions considered the expected value is nearly identical for $\sigma \geq 0.5 \mathrm{~V}_{\text {rms }}$. Also the differences between distributions disappear as noise power increases. So the actual function is considerably robust to the noise distributions. However differences arise when the error or standard deviation is considered. In general the soft limiter has lower values of variance and uniform distribution has lower levels for low noise power but higher for large noise compared to Gaussian noise.

\subsection{Modelling arrays of gates}

Once the individual output characteristics are determined we address the array system $(n>1)$. When the array is considered there are two effects to consider. The first is that by combining all the individual outputs into the global output, $\bar{y}$, the variance is modified, and second that the global output is a multivalued signal with as many a $2^{n}$ different levels (both are dependant on the correlation among $y_{i}$ signals). These facts can be seen if the pdf is worked out. However it is difficult to derive it and usually only numerical approaches can be used. Besides we are interested in the system function and error. In general (i.e. without considering any limitations to the random variables $y_{i}$ ) we can calculate the expected value conditioned to the input variable $x$ and its variance as the sample mean and sample variance of the random array composed by all individual outputs as

$$
E\{\bar{y}\}=\frac{1}{n} \sum_{i=1}^{n} E\left\{y_{i}\right\},
$$



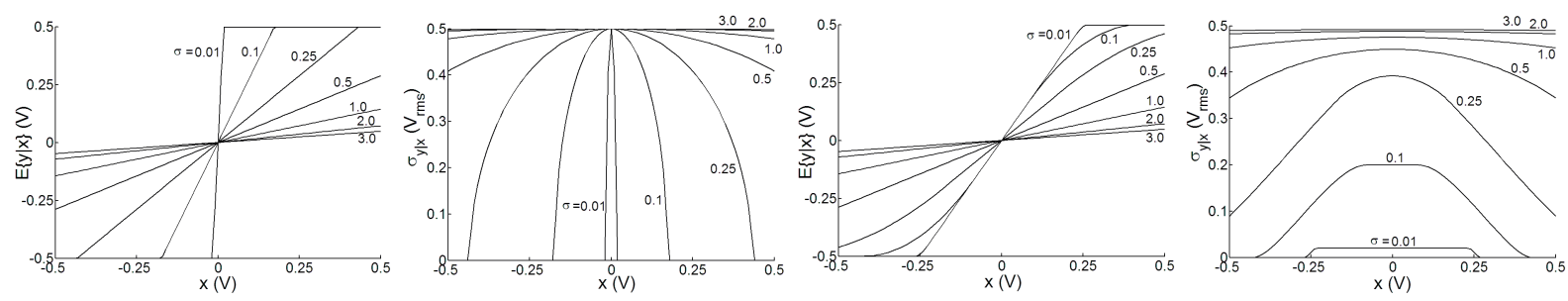

Figure 5. Expected value and standard deviation of a single gate output for a hard limiter (left) and soft limiter (right) driven by a uniform noise with standard deviation ranging from $0.01 \mathrm{~V}_{\text {rms }}$ to $3.0 \mathrm{~V}_{\text {rms }}$. The gates have $a_{h}=0.5 \mathrm{~V}$ and $a_{l}=-0.5 \mathrm{~V}$, and thresholds $b=0 \mathrm{~V}$ for the hard limiter and $b_{h}=0.25 \mathrm{~V}$ and $b_{l}=-0.25 \mathrm{~V}$.
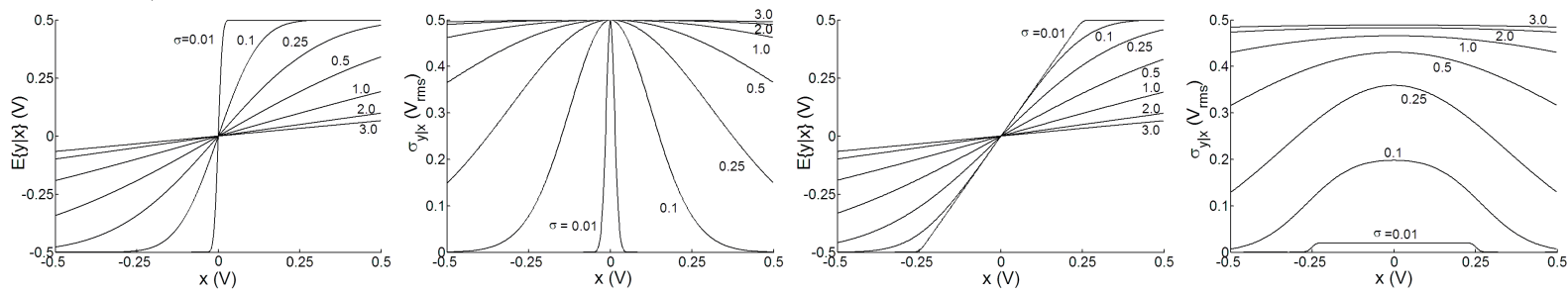

Figure 6. Expected value and standard deviation of a single gate output for a hard limiter (left) and soft limiter (right) driven by Gaussian noise with the same configuration as Fig. 5.

$$
\sigma_{\bar{y}}^{2}=\frac{1}{n^{2}} \sum_{i=1}^{n} \sum_{j=1}^{n} r_{i j} \sigma_{x_{i}} \sigma_{x_{j}},
$$

where $r_{i j}$ is the correlation coefficient between output $y_{i}$ and $y_{j}$. If we consider the structure under study we can see that all individual signals have the same distribution. So the global expected value is equal to the individual

$$
E\{\bar{y}\}=E\left\{y_{i}\right\}
$$

The variance also simplifies if the signals are uncorrelated or i.i.d as

$$
\sigma_{\bar{y}}^{2}=\frac{1}{n^{2}} \sum_{i=1}^{n} \sigma_{y_{i}}^{2}=\frac{\sigma^{2}}{n} .
$$

So in general the array system performs the same function as the individual cells, but with a lower error that decreases as the number of parallel gates increases.

\section{COMPARISON BETWEEN MODEL DATA AND SIMULATED DATA: STOCHASTIC RESONANCE IN NON-IDEAL COMPARATORS}

The model is useful to study both noise and fluctuations on process parameters on the gates. However due to space limitations we will show only effects due to noise, in this paper. In this section, we present a comparison between the predicted form of the model and simulation data. We consider a sinusoidal signal as input for the gates and both uniform and Gaussian white noise.

Two different cases are presented. We consider two sinusoid signals with frequency $10 \mathrm{~Hz}$ and amplitude $0.2 \mathrm{~V}$, but one has an offset of $-0.5 \mathrm{~V}$ and the other a null offset. So both effects (subthreshold and suprathreshold SR are considered). The input signal is modelled statistically by its pdf as

$$
f_{x}(x)=\frac{1}{\pi \sqrt{x_{\mathrm{amp}}^{2}-\left(x-x_{\mathrm{off}}\right)^{2}}},
$$

where $x_{\mathrm{amp}}$ is the amplitude and $x_{\mathrm{off}}$ the offset of the sinusoid. 


\subsection{Signal to noise ratio in time domain}

To be able to compare simulations and model predictions it is necessary to use a measure of performance. Several different measurements have been proposed for quantifying SR. ${ }^{12,20,21,23}$ We use the SNR calculated in the time domain ${ }^{24}$ as is well-known in engineering, which only uses temporal information. In general the SNR is the ratio between signal and noise powers in decibels. So we must calculate both the power for the output signal and for the noise for the analytical and simulated data.

$$
\mathrm{SNR}=10 \log \left(\frac{P_{S}}{P_{N}}\right)
$$

\subsubsection{Analytical data}

From the description of the systems under study we have obtained the expected value conditioned to the input signal and the variance conditioned also to the input value. Output signal power is proportional to the square value of the output once its continuous value is removed. We can calculate this value as

$$
P_{S}=\int_{x}(E\{y \mid x\}-E\{y\})^{2} f_{x}(x) d x=\int_{x} E^{2}\{y \mid x\} f_{x}(x) d x-E^{2}\{y\} .
$$

The noise power is simply the variance of the output

$$
P_{N}=\sigma_{y}^{2}=\int_{x} \sigma_{y \mid x}^{2} f_{x}(x) d x=\int_{x} \int_{y} y^{2} f_{y}(y \mid x) d y f_{x}(x) d x-\int_{x} E^{2}\{y \mid x\} f_{x}(x) d x .
$$

\subsubsection{Simulation data}

To work out signal power from the temporal series it is necessary to use averages along sets of runs to obtain the mean value at each instant of time and then subtract the temporal average. The same procedure was used in. ${ }^{24}$

$$
P_{S}=\left\langle(\bar{y}-\langle\bar{y}\rangle)^{2}\right\rangle
$$

where the overbar denotes ensemble average and the angle brackets denote temporal average. The noise power is calculated as the mean along the runs of the mean error of each run to ensemble mean,

$$
P_{N}=\overline{\left\langle(y-\bar{y})^{2}\right\rangle}
$$

\subsubsection{Stochastic resonance in a single gate}

We consider two gates with with outputs switching from $a_{l}=-0.5 \mathrm{~V}$ and $a_{h}=0.5 \mathrm{~V}$. One being a hard limiter with threshold set to $b=0 \mathrm{~V}$ and the other a soft limiter with a transition from $b_{l}=-0.25$ to $b_{h}=0.25 \mathrm{~V}$. Figure 7 shows the plots for these gates for both input signals (subthreshold and suprathreshold) considering uniform and Gaussian noise. Continuous lines are for the simulated data while the points are from the theoretical equations ( + for uniform noise and $\times$ for Gaussian). The agreement is satisfactory.

The plots show that uniform noise produces a higher SNR resonance peak, but the performance degrades quicker than Gaussian noise. Independently from the noise distribution considered, the soft limiter gate has a resonant peak around $5 \mathrm{~dB}$ higher than the hard limiter and the peak appears for lower noise intensities. As expected, there are no resonant peaks when suprathreshold signals are considered with a single gate. As seen from Figures 5 and 6, the behaviour of all systems converge to the same response for large noise values. 

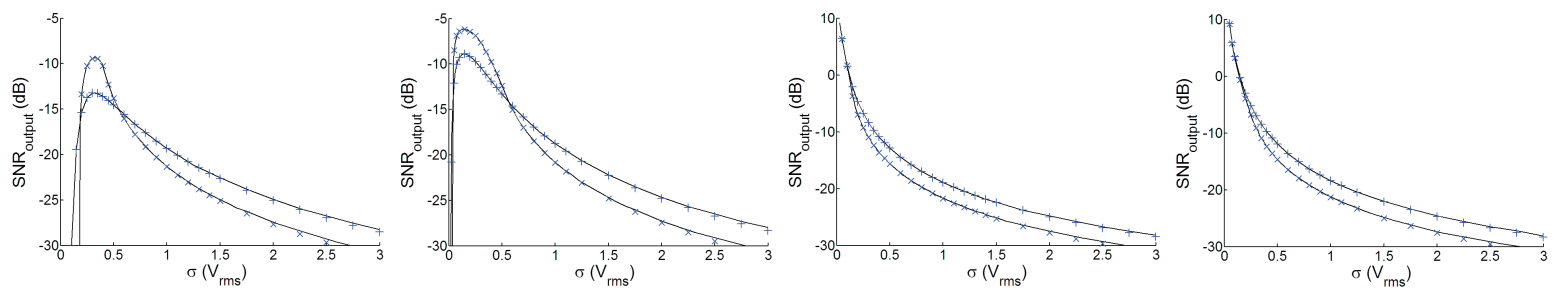

Figure 7. SR plots for the hard limiter (HL) and soft limiter (SL). Left: Subthreshold input signal (offset $=-0.5 \mathrm{~V}$ ) for the HL and SL correspondingly. Right: Suprathreshold input signal (offset $=0 \mathrm{~V}$ ) for HL and SL. Continuous lines correspond to simulated data and symbols to predicted results from the model ( + for uniform noise and $\times$ for Gaussian).
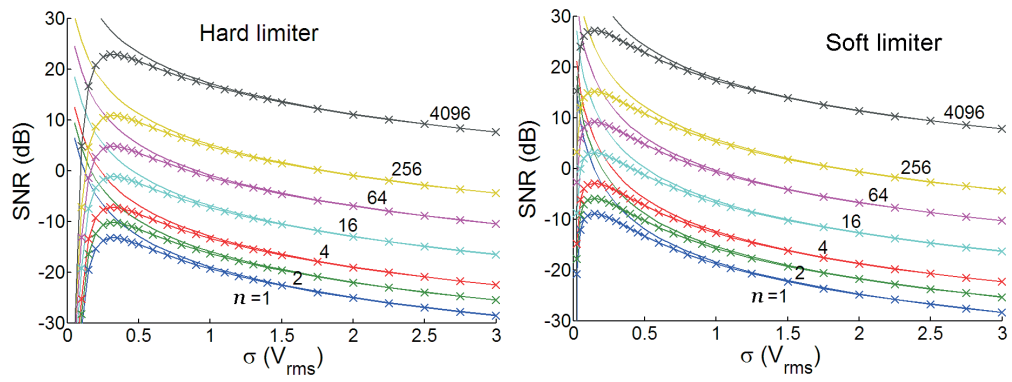

Figure 8. SNR plots for subthreshold and suprathreshold input signal and Gaussian noise for array systems with $n=1,2$, 4, 16, 64, 256 and 4096 gates: - for suprathreshold input signals, $\times$ for subthreshold input signals. Using this measure it is clear that the only difference between a single gate and an array of them is the averaging. No changes on the behaviour can be seen.

\subsubsection{Stochastic resonance in an array system}

As shown in Section 2.2 the array system performs the same function as the single gate, but with a reduction in the variance proportional to $1 / n$ by both the increase in degrees of freedom (possible values in the output) and the effect of the averaging or combinations of uncorrelated noise. Figure 8 depicts the plots for the hard limiter and soft limiter gates for arrays of $n=1,2,4,16,64,256$ and 4096 for Gaussian noise (uniform noise is not presented as the comparison presented for a single gate remains valid for the array system). Suprathreshold and subthreshold input signals are shown.

As stated $i^{24}$ there is no resonance when suprathreshold signals are considered. This is caused by the fact that the measurement of SNR, in the time domain, considers as signal power the ensemble average. This is correct, but the result is that the increase in the degrees of freedom at the output is not taken into consideration. For this measure a single bit output is as good as a 10-bit output. The result is that only difference between a single gate and the array of gates is the variance reduction by the effect of the averaging. The question of SR described by this measure is closely related to the detectability problem in optimal or suboptimal detectors. ${ }^{25}$

\subsection{Modified signal to noise ratio in time domain}

One important reason for using SNR in the time domain is that it is valid for non-linear systems, where the input signal usually is profoundly changed by the system. In these systems measurements based on linear systems are not suitable. However as seen in the previous section, SNR in the time domain does not capture all the effects that noise induces in an array of gates. In this section we propose a modified version of the SNR that keeps the advantages of the time domain being suitable for non-linear systems, but that takes into account all effects of noise. This measure calculates the signal power, as stated in Eq. (28), but the noise power is slightly modified. We propose to consider noise power as comprising two factors. One is the variance, as in the original measure. 

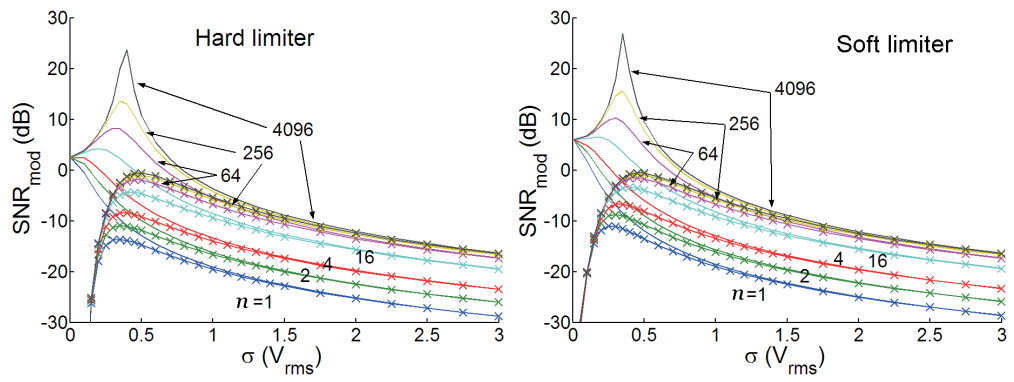

Figure 9. Modified SNR Plots for subthreshold and suprathreshold input signal for an array system with $n=1,2,4$, 16, 64, 256 and 4096 gates and Gaussian noise: - for suprathreshold input signals, $\times$ for subthreshold input signals.

The other is the error between the expected value of the output and the desired function, $h(x)$, without their offsets. So the noise power is

$$
P_{N}=\int_{x}\left([(h(x)-E\{h(x)\})-(E\{y \mid x\}-E\{y\})]^{2}+\sigma_{y \mid x}^{2}\right) f_{x}(x) d x .
$$

This measure is interesting as it relies on time domain information and so it is useful for non-linear systems - it relates the system performance with a target function of any sort and we can calculate the array response easily from the simple single gate description without using the actual pdf for the global output.

Using the modified SNR and considering $h(x)=x$ for the results in Figure 8, we can see in Fig. 9 that it is in fact resonance for both suprathreshold and subthreshold signals. This measure shows the same characteristics seen in Fig. 8. However there some important differences. In this SNR measure it is clear that when large noise is present there is a maximum amount of information that can be recovered, even if we increase the number of gates in the array. The peak of the suprathreshold SR curve grows with the number of gates, as all the extra degrees of freedom are used to improve the signal. However, when subthreshold SR is considered it saturates the resonant peak. This is due to the fact that the input signal only covers a portion of the input range that noise defines.

\section{DISCUSSION}

As shown in this work, and in existing SR literature, when noise is present in non-linear systems it assists the flow of information through the system. In some cases, the effects are maximized when noise and input signal have a similar amplitude (around $0 \mathrm{~dB}$ of SNR as reported for sensory neurons ${ }^{7}$ ). Furthermore, as Figures 5 and 6 show, noise changes the system transfer function by adjusting the resulting gain to map the input noise over the output range. This means that if noise is considered, any input range can be mapped onto the output range without changing any characteristics of the circuits, only the noise amplitude.

The structure of an array of identical gates provides one of the best ways to deal with defects. ${ }^{26}$ In fact, this structure provides the redundancy necessary to be tolerant to defects, moreover it uses all the gates available to improve the system response in the best possible way. One frequent question is to consider that by setting all the thresholds to an equal value, the design is not optimal. However as Figure 10 shows when noise and signal have comparable power it is the optimal design. ${ }^{27}$ At the same time, by considering noise as a part of the system the concept of fault tolerance is inherent in the design. The variance of the output can be adjusted to the levels demanded by the application.

All these show that by considering noise in the design process we can obtain electronic gates that can work with extremely low levels (in the same amplitude range than the noise) which will reduce the electric fields and the power dissipation. Also more relaxed characteristics for the gates can be allowed, as noise will reshape their transfer functions permitting a larger variation in process parameters. Finally the redundancy is used for taking 

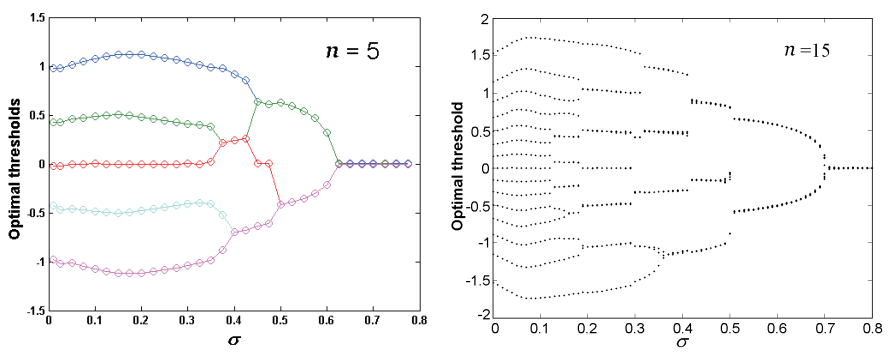

Figure 10. Optimal threshold values to maximize information transference for an array of $n=5$ and $n=15$. Here, $\sigma$ is the ratio between noise and signal standard deviations $\left(\sigma_{\eta} / \sigma_{x}\right)$. As noise intensity approach signal the optimal threshold values tends to group all together to the mean value (in this case zero). See McDonnell ${ }^{27}$ for more detail.

care of defects and faults, but without useless elements as all of them work for the system - allowing a way to deal with the yield reduction expected in future nanoelectronic technologies.

The use of noise in an array structure is quite interesting, but there are some questions that must be considered. First, adding noise is a useless power consuming action, so what is interesting is using internal system noise or inherent fluctuations. That means the devices must be sensitive enough to work at those levels. Second, gates are dynamical systems and so they require a dynamical model to derive the relations between signal, noise and gate in the temporal or frequency domains. And third, the noise considered in this and most SR work is i.i.d. noise. If electronic noise is considered without any further consideration, the SNR peaks are greatly reduced, ${ }^{22}$ in actual electronic chips, due to the substrate and the proximity of the gates. This proximity means that the noise in each element is nearly identical.

\section{CONCLUSION}

A simple model valid for predicting both noise and process deviations in gates is presented. The extension to an array of gates is straightforward, being of interest for predicting the response in large parallel ensembles. Using the model we describe both hard limiter and soft limiter gates, with uniform and Gaussian white noise. The combined function of gate and noise is studied showing that due to noise the resulting function converges to the same input-output relation, as noise grows independently of either the circuit function or the noise distribution. The error or variance of the output is also shown. We can see that soft limiter circuits have a lower variance value near the threshold values than hard limiter gates.

To check the predictions of the model, we study SR plots for both subthreshold and suprathreshold signals. We measure the SR using the SNR in the time domain showing that the agreement between model and simulation is very good. Using these results we study the SR when non-ideal comparators are considered showing that in general the stochastic resonance peaks for soft limiter circuits are higher than that from hard limiters. Finally we propose a modified SNR measure, which takes into account all the effects that noise produces in an array system. Using this measure it is possible to see both subthreshold and suprathreshold SR. Furthermore, this modified SNR shows that for large noise values the information transfer cannot be improved indefinitely by adding more elements in the array. Also we see that the stochastic resonant peak for subthreshold SR saturates, due to the loss of levels for describing the offset of the input signal, while the peak always improves with the number of elements in the array for suprathreshold SR.

\section{ACKNOWLEDGMENTS}

This work has been supported by the grant AP2002-2600 and the project CYCIT TIC 2001-2337. Funding from GTECH Australasia is also gratefully acknowledged. 


\section{REFERENCES}

1. "International technology roadmap for semiconductors," http://public.itrs.net/Files/2003ITRS/Home.htm, 2003.

2. K. Nikolic, A. Sadek, and M. Forshaw, "Fault-tolerant techniques for nanocomputers," Nanotechnology 413 , pp. 357-362, 2002.

3. M. N. Mihaila, "Low-frequency noise in nanomaterials and nanostructures," in Noise and fluctuations control in electronic devices, A. A. Balandin, ed., pp. 367-385, American Scientific Publishers, Los Angeles, 2002.

4. D. Challet and N. F. Johnson, "Optimal combinations of imperfect objects," Phys. Rev. Lett. 89, 028701, 2002.

5. J. K. Douglass, L. Wilkens, E. Pantazelou, and F. Moss, "Noise enhancement of information transfer in crayfish mechanoreceptors by stochastic resonance," Nature 365, pp. 337-340, 1993.

6. J. E. Levin and J. P. Miller, "Broadband neural encoding in the cricket cercal sensory system enhanced by stochastic resonance," Nature 380, pp. 165-168, 1996.

7. M. DeWeese and W. Bialek, "Information flow in sensory neurons," Il Nuovo Cimento 17D, pp. 733-741, 1995.

8. X. Yu and E. R. Lewis, "Studies with spike initiators: linearization by noise allows continuous signal modulation in neural networks," IEEE Trans. on Biomedical Eng. 36, pp. 36-43, 1989.

9. B. Ando and S. Graziani, "Adding noise to improve measurement," IEEE Instr. and Meas. Magazine 4, pp. 24-31, 2001.

10. R. W. Stewart and E. Pfann, "Oversampling and sigma-delta strategies for data conversion," Elec. and Com. Eng. Jour. 10, pp. 37-47, 1998.

11. S. Meninger, J. O. Mur-Miranda, R. Amirtharajah, A. Chandrakasan, and J. H. Lang, "Vibration-to-electric energy conversion," IEEE Trans. on VLSI Systems 9, pp. 64-76, 2001.

12. F. Chapeau-Blondeau and D. Rousseau, "Noise improvements in stochastic resonance: from signal amplification to optimal detection," Fluctuation and Noise Lett. 2, pp. L221-L233, 2002.

13. A. A. Saha and G. V. Anand, "Design of detectors based on stochastic resonance," Signal Processing $\mathbf{8 3}$, pp. 1193-1212, 2003.

14. A. Nikitin, N. G. Stocks, and A. R. Bulsara, "Nonlinear signal detection in the time domain: level crossing statistics and noise-mediated minimization of the measurement error," Fluctuation and Noise Lett. 4, pp. L63-L73, 2004.

15. I. Y. Lee, X. Liu, B. Kosko, and C. Zhou, "Nanosignal processing: stochastic resonance in carbon nanotubes that detect subthreshold signals," Nano Letters 3, pp. 1683-1686, 2003.

16. J. C. Comte and S. Morfu, "Stochastic resonance: another way to retrieve subthreshold digital data," Phys. Lett. A 309, pp. 39-43, 2003.

17. G. G. Karapetyan, "The increase of gravitational-wave interferometer sensitivity by employing stochastic resonance mechanism in nonlinear white-light cavity," Optics Communications 238, pp. 35-43, 2004.

18. N. G. Stocks, D. Allingham, and R. P. Morse, "The application of suprathreshold stochastic resonance to cochlear implant coding," Fluctuation and Noise Lett. 2, pp. L169-L181, 2002.

19. G. P. Harmer and D. Abbott, "Motion detection and stochastic resonance in noisy environments," Microelectronics Journal 32, pp. 959-967, 2001.

20. N. G. Stocks, "Suprathreshold stochastic resonance in multilevel threshold systems," Phys. Rev. Lett. 84, pp. 2310-2313, 2000.

21. M. D. McDonnell, C. E. M. Pearce, and D. Abbott, "A characterization of suprathreshold stochastic resonance in an array of comparators by correlation coefficient," Fluctuation and Noise Letters 2, pp. L205-L220, 2002.

22. F. Martorell, M. D. McDonnell, D. Abbott, and A. Rubio, "Generalized noise resonance: Using noise for signal enhancement," in Proc. SPIE Fluctuations and Noise in Biological, Biophysical, and Biomedical Systems II, D. Abbott, S. M. Bezrukov, A. Der, and A. Sanchez, eds., 5467, pp. 163-174, 2004.

23. L. Gammaitoni, P. Hanggi, P. Jung, and F. Marchesoni, "Stochastic resonance," Reviews of Modern Physics 70, pp. 223-287, 1998. 
24. N. G. Stocks, "Information transmission in parallel threshold arrays: Suprathreshold stochastic resonance," Phys. Rev. E 63, 041114, 2001.

25. S. Kay, "Can detectability be improved by adding noise?," IEEE Signal Processing Lett. 7, pp. 8-10, 2000.

26. A. Schmid and Y. Leblebici, "Robust circuit and system designmethodologies for nanometer-scale devices and single-electron transistors," IEEE Transactions on Very Large Scale Integration (VLSI) Systems 12, pp. 1156-1166, 2004.

27. M. D. McDonnell, N. G. Stocks, C. E. M. Pearce, and D. Abbott, "Point singularities and suprathreshold stochastic resonance in optimal coding," arXiv:cond-mat/0409528, 2004. 